\title{
LAS INTERACCIONES DIDÁCTICAS EN EL MARCO DE LAS PRÁCTICAS EDUCATIVAS EN LA VIDEOCONFERENCIA
}

\author{
Dr. Alejandro Armellini** \\ D.E. Mirian Gregori** \\ D.E. Lely Núñez Coronel *** \\ D.E. Amadeo Sosa****
}

\section{Introducción}

En el último siglo los avances científicos y tecnológicos fueron objeto de una mayor rapidez de cambio que en los siglos anteriores. Esto tiene su repercusión en lo económico, social, educativo, así como en tantas otras áreas, planteando la necesidad de una formación ciudadana básica que le permita a los individuos reconocer e interpretar los cambios que se están produciendo a su alrededor. Esto exige de los docentes una formación permanente y de sus alumnos, la necesidad de aprender a seleccionar, jerarquizar y utilizar la información que reciben a través de los nuevos medios tecnológicos. Por otra parte, es necesario superar la distancia para lograr una actualización continua de los conocimientos de los profesionales, particularmente en regiones distantes de los centros principales de actividad educativa, como en el interior de Uruguay, donde es difícil acceder a la capacitación buscada. A partir de la Revolución Industrial, se comenzaron a implementar estrategias de Educación a Distancia (EaD) aún vigentes, que permiten una difusión masiva, a través de materiales impresos y posteriormente por la radio y la TV. En la actualidad, nuevos medios han aparecido por lo que es posible además, conocer al docente y a los "compañeros de clase" e interactuar con ellos a través de la videoconferencia (VC). Esta herramienta es utilizada para aplicaciones que requieren interacción en tiempo real y comunicación visual entre dos o más sitios geográficamente distantes.

En el ámbito educativo cada vez que se introduce una nueva herramienta tecnológica se tiende, en general, a realizar una asimilación deformante de la misma, es decir se utiliza ésta con las prácticas pedagógicas anteriores. Posteriormente, con el uso y aplicación se van haciendo las correcciones necesarias, dejando de lado algunas técnicas, estrategias o procedimientos, a veces creando nuevos y otras adaptándolos, hasta que surgen investigaciones y estudios sistemáticos que permiten un mejor aprovechamiento educativo.

\section{Objetivos de la investigación}

Se pretende abrir interrogantes, e iniciar líneas de investigación que hagan posible una adecuación de los procesos de enseñanza existentes a la $\mathrm{VC}$, potenciando desde el punto de vista pedagógico su utilización. Procuraremos describir e ilustrar las interacciones que deliberadamente provoca el docente, en este nuevo espacio educativo. Pretendemos además determinar las prácticas pedagógicas que favorecen las interacciones didácticas en la VC. Por todo esto nos planteamos la siguiente pregunta: ¿Qué características tienen las interacciones didácticas que se producen en las clases por VC y qué prácticas pedagógicas las fomentan? 
Capítulo 4 Las interacciones didácticas en el marco de las prácticas educativas en la videoconferencia

\section{Objetivo general}

Contribuir al mejoramiento de la enseñanza impartida a través de la videoconferencia.

\section{Objetivos específicos}

Identificar las características de las interacciones didácticas que se producen en la videoconferencia.

Observar las prácticas pedagógicas que favorecen las interacciones didácticas en la videoconferencia.

\section{Marco teórico}

\section{Educación a distancia}

La Educación a Distancia (EaD) es un hecho de indudable significación social, económica, cultural y política, que cada día alcanza a mayor número de países, centros educativos y alumnos. Se puede entender la $\mathrm{EaD}$ como la provisión de un ambiente de aprendizaje a un alumno remoto, por lo que la única diferencia real entonces es que la mayor parte de la comunicación entre profesor y alumno es mediada. Serrano (1998) la define como "Modalidad educativa que comprende una situación formal de enseñanza y de aprendizaje donde el docente y el discente, se encuentran en una dimensión témporoespacial distinta, debiendo por ello establecer una relación a través de diferentes medios y modelos de comunicación, de tal forma que facilite la transmisión y la recreación del conocimiento, con posibilidad de diálogo e interacción síncrona o asíncrona".

Actualmente, la función más importante de los medios en EaD es como canal de comunicación que permite la interacción de profesores y alumnos, ya sea sincrónica (que sucede en el mismo tiempo) o asincrónica (en diferente tiempo). Por esta razón es importante que todos los involucrados en el acto educativo conozcan bien las ventajas y limitaciones que el medio que utilizarán les impone, para lograr de esta forma una comunicación eficaz.

\section{La videoconferencia}

La VC es una tecnología relativamente nueva, surgida alrededor de 1980, en la cual una comunicación bidireccional y sincrónica de imagen, sonido y datos permiten la interacción entre dos o más sitios geográficos distantes. Justamente esa posibilidad de interacción es una de las características principales de este medio, por lo que no se debe interpretar a la VC como una "conferencia por video", sino como un encuentro virtual, entre personas que están distantes geográficamente, para comunicarse entre sí. Se utiliza para aplicaciones que requieren interacción en tiempo real y comunicación visual, por lo que los teleasistentes pueden verse y oírse como si estuvieran en la misma clase.

Cabe preguntarnos si su uso como herramienta educativa constituye efectivamente una forma de educación a distancia. Resulta evidente que esta herramienta permite que personas geográficamente distantes de los centros de estudio puedan acceder a cursos y enriquecer su formación. La comunicación e interacción es bidireccional y en tiempo real. Sin embargo, el uso de esta herramienta exige la presencia de alumnos y docentes en lugares determinados (salas de videoconferencia) en momentos establecidos (el horario de clase). Es por este motivo que hay autores que utilizan el término «telepresencia», cuyo significado literal es «presencia remota», siendo la videoconferencia interactiva uno de los sistemas que la facilitan (GATE, 1998: 12-13).

46 - Universidad ORT Uruguay 
Existen obstáculos para una implementación adecuada de la $\mathrm{VC}$, algunos relacionados con el aspecto tecnológico y otros con el aspecto pedagógico. Entre los primeros cabe destacar: las diferentes arquitecturas de hardware; el limitado ancho de banda para la transmisión de información; la baja calidad de las imágenes; la falta de sincronización entre la voz y la imagen; los altos costos que implican los cambios y que inevitablemente imponen límites a la cantidad de sitios y accesos.

En el aspecto pedagógico se destacan: las dificultades debidas a la asincronización mencionada anteriormente, que pueden impedir una interpretación crítica de la información recibida (Tiffin y Rajassingham, 1995); la necesaria preparación de los docentes que el uso de la VC requiere, por los distintos aspectos tecnológicos involucrados en la misma (Universidad de Indiana, 1996); la falta del "cara a cara" tan importante en una relación pedagógica y la ausencia de técnicos de producción artística que tengan participación en el mejoramiento de la imagen y dicción, evitando así que la videoconferencia de hoy guarde similitudes con una versión a color de la TV de hace cincuenta años (cf Latchem, 1995: 100).

Sobre la utilización de la VC en educación, Galindo nos dice: "Hay que destacar que lo que convierte en educativo a un medio, es justamente la planeación y el diseño didáctico que se empleen en su elaboración. Los medios por sí mismos no son instrumentos de aprendizaje, es la intencionalidad de enseñar algo y la estrategia aplicada para lograrlo, que los convierten en recurso educativo" (1998: N²). La efectividad, entonces, de la enseñanza por VC parece depender más del aspecto pedagógico que de las características inherentes al medio.

\section{Interacciones didácticas}

La interacción, tal como la ha definido Postic, es "la relación recíproca, verbal o no verbal, temporal o repetida según una cierta frecuencia, por la cual el comportamiento de uno de los interlocutores tiene una influencia sobre el comportamiento del otro". (1982: 84) La interacción es considerada entonces, como la acción de influencia y reciprocidad que se establece entre dos o más sujetos en un entorno definido. Cuando este entorno es el aula, los sujetos intervinientes son el profesor y los alumnos, en cuanto a su rol en la interacción. En el docente inciden sus concepciones de enseñanza y de aprendizaje, las características del grupo, del contexto social, entre otros. En el alumno influyen las percepciones que tiene del docente, de la enseñanza y de sí mismo, así como también la influencia de sus compañeros, del contexto social, etc. Por ello el análisis de la interacción en el aula ha sido un campo de investigación preferente para pedagogos, psicólogos y sociólogos, y una preocupación constante en los paradigmas y modelos de investigación sobre enseñanza.

La interacción didáctica "es un proceso comunicativo-formativo, caracterizado por la bidireccionalidad y reciprocidad de los agentes participantes en ella. La interacción se incorpora a la enseñanza, siendo más que una comunicación o influencia mutua, una fuerza cohesionadora que hace eficiente el proceso de enseñanza-aprendizaje, en cuanto sirve al alumno para adquirir una formación intelectual o actitudinal" (Medina, 1989: 31).

En el contexto de la enseñanza por videoconferencia, las interacciones se ven afectadas por las posibilidades y limitaciones de este medio tecnológico. Puede argumentarse que la ventaja del mismo radica en la sincronía que permite la interacción entre docente y alumnos y lo convierte en el medio más cercano al trabajo en el aula, por lo que puede verse como un medio similar a su entorno habitual de enseñanza (GATE, 1998: 19). En contrapartida, la relación didáctica se ve afectada frecuentemente por la tecnología y depende de diversos elementos como la conexión, la imagen, el audio, la cantidad de sedes conectadas y la habilidad del docente para crear situaciones de interacción, entre otros. 
Capítulo 4 Las interacciones didácticas en el marco de las prácticas educativas en la videoconferencia

\section{Prácticas pedagógicas}

Las prácticas pedagógicas están relacionadas con el quehacer docente en el aula. Son "aquellas mediante las cuales los docentes facilitan, organizan y aseguran un encuentro y un vínculo entre los alumnos y el conocimiento, y están estrechamente vinculadas con la transposición didáctica". (Bengoa y González, 1998: 23) Son observables en las distintas intervenciones docentes, en la acción que desarrollan en la clase y a través de la cual procuran el aprendizaje de sus alumnos. En consecuencia, dichas prácticas, están vinculadas a las metodologías y técnicas que desarrolla el docente en forma explícita o implícita, en un medio tecnológico que exige por lo menos una revisión y análisis sobre la adecuación y pertinencia de las mismas.

El auge creciente de los enfoques cognitivos ha llevado a destacar el carácter constructivo del proceso de adquisición del conocimiento. La idea del alumno fácil de moldear ha sido poco a poco sustituida por la del estudiante que selecciona, asimila, procesa, interpreta y da significado a los contenidos. En el campo educativo este cambio de perspectiva ha contribuido, por una parte, a poner de relieve lo inadecuado de unos métodos de enseñanza esencialmente expositivos que conciben al profesor y al alumno como simples transmisor y receptor de conocimientos respectivamente y, por otra, a revitalizar las propuestas pedagógicas que sitúan en la actividad autoestructurante del alumno, el punto de partida necesario para un verdadero aprendizaje. La ambigüedad del papel del profesor en algunas propuestas pedagógicas que consideran la actividad autoestructurante del alumno como el factor decisivo, único y determinante del aprendizaje, no tienen en cuenta la interacción sistemática entre los actores del proceso educativo, alumnos y profesor. Por esto no se debe entender el acto educativo como un proceso exclusivamente de aprendizaje, sino que debe existir también una voluntad manifiesta del profesor de incidir sobre dicho aprendizaje, tomando una serie de decisiones sobre lo que tiene que aprender el alumno y sobre las condiciones óptimas para que lo aprenda (Coll, 1994: 133-136).

En la clase por VC aparece una distancia, entre el docente y los alumnos, que trasciende a la distancia geográfica y que está relacionada con una interacción mediatizada por una pantalla, donde por un lado se encuentran los alumnos, y por otro, el profesor. Es a través de esa pantalla que tienen lugar los procesos de enseñanza y de aprendizaje. Las prácticas pedagógicas utilizadas por los docentes en este medio se constituyen entonces en elementos claves de observación, pues los profesores además de seleccionar los contenidos y la metodología que utilizarán para acercar el conocimiento a los alumnos, también deberán adecuarlos a este medio, resolviendo los problemas y dificultades que puedan surgir de esta interacción mediatizada. Lograr la participación de los alumnos adquiere, entonces, una relevancia particular, tanto como la del profesor en la presentación de los saberes.

\section{Diseño metodológico}

Se optó por un enfoque de corte cualitativo. Esto obedece a que esta investigación procuró analizar las interacciones didácticas provocadas por el docente en la enseñanza por $\mathrm{VC}$, partiendo de la información que arrojan los datos. Se abarcó la totalidad de los cursos por VC que se realizaron en la Universidad ORT Uruguay, de setiembre a diciembre de 1998.

\section{La investigación fue de tipo:}

- Exploratoria, debido a que las interacciones didácticas y las prácticas pedagógicas que las fomentan en la videoconferencia han sido poco exploradas, ya que se trata de la aplicación de una herramienta tecnológica bastante reciente.

- Descriptiva, por cuanto se trató de describir las características fundamentales de las interacciones didácticas que ocurren en las clases por videoconferencia.

48 - Universidad ORT Uruguay 
Capítulo 4 Las interacciones didácticas en el marco de las prácticas educativas en la videoconferencia

De acuerdo con el enfoque de nuestra investigación los instrumentos seleccionados fueron los siguientes:

Observación directa y no participante de clases, tanto desde la sala de emisión como desde los sitios remotos. El propósito de estas observaciones fue obtener una descripción detallada de las interacciones didácticas y las prácticas pedagógicas que las generan, en el entorno de la videoconferencia.

Observación de las clases grabadas en video. Se pudo apreciar que a través de las observaciones de los videos, la calidad y cantidad de los datos obtenidos eran mejores, debido a las características del aula de emisión, ya que desde nuestra ubicación sólo se podía observar al profesor. En cambio, observando los videos se apreciaban los alumnos en las distintas sedes, y se eliminaba el inconveniente principal de este instrumento, las alteraciones o modificaciones que pueden realizar los observados ante nuestra presencia durante su clase.

Entrevistas por pautas a docentes, para establecer la percepción de éstos acerca de la enseñanza por videoconferencia y determinar posibles diferencias con su experiencia en escenarios presenciales.

Cuestionarios aplicados a los alumnos de las cuatro sedes, con el objetivo de acercarnos a su percepción sobre la situación de enseñanza en la videoconferencia.

También realizamos numerosas notas de campo durante todo el desarrollo de la investigación y se utilizó el correo electrónico como estrategia de campo para la coordinación y comunicación de novedades.

\section{Análisis de datos}

El análisis de los datos se fue realizando a medida que se iban obteniendo éstos de las distintas fuentes, codificándolos para garantizar el anonimato de las fuentes. Desde el comienzo se fueron elaborando las categorías en base a la comparación de los registros e informaciones obtenidas.

En el discurso comunicativo utilizado en el aula ocurren múltiples interacciones. En un primer análisis de los datos obtenidos en las observaciones, las interacciones se diferenciaron en función de atributos o características comunes, categorizándolas en didácticas y no didácticas. En una segunda instancia se estableció un sistema de categorías dentro de las interacciones didácticas en el cual se identificaron dos grupos: dialécticas y no dialécticas.

Dadas las características del tema investigado, la aproximación cualitativa al mismo, y los niveles exploratorios y descriptivos que se manejaron, se dividió el análisis en tres grandes partes para facilitar su estudio. En primer lugar se analizaron las interacciones didácticas encontradas en los cursos por VC. En segundo lugar se desarrollaron algunos factores que influyen en las interacciones. Por último se vieron las prácticas pedagógicas que las favorecían.

\section{Interacciones dialécticas}

A los efectos de nuestro trabajo, consideramos necesario precisar aun más el alcance de lo que constituye una interacción didáctica. En la enseñanza pueden darse procesos comunicativo-formativos que debido a la ausencia de una influencia mutua entre los actores no constituyen procesos interactivos desde lo observable. Por esto la exposición en el aula no implica, a nuestro criterio, una interacción didáctica, ya que no podemos observar la bidireccionalidad y reciprocidad entre el docente y los alumnos en los aspectos comunicativos centrados en el objeto de conocimiento. Para que una interacción sea didáctica, además de una influencia mutua entre los actores, es necesario que se generen espacios de discusión, de análisis, de reflexión que procuren la aclaración de un concepto, la adquisición de cierta idea o de cierto conocimiento. 
Capítulo 4 Las interacciones didácticas en el marco de las prácticas educativas en la videoconferencia

En nuestro análisis nos concentramos específicamente en las interacciones que consideramos didácticas, a las que categorizamos en dialécticas y no dialécticas tomando como criterio las formas de acercar el conocimiento al alumno.

\section{Interacciones dialécticas}

Las interacciones dialécticas responden a una intervención intencional del profesor a través de preguntas relacionadas entre sí y con las respuestas dadas, para que los alumnos como resultado de las mismas, alcancen el conocimiento en el sentido de reelaborarlo y relacionarlo con conocimientos anteriores. Las características de estas interacciones están dadas por: un sistema de preguntas y respuestas alrededor de una cierta temática, el coprotagonismo en la elaboración conceptual, una búsqueda cooperativa del conocimiento y por el énfasis en la construcción de significados. Para ello, el docente diseña en forma deliberada y consciente ciertas actividades, cuyo fin último es que el alumno adquiera, un nuevo conocimiento o habilidad.

\section{Interacciones no dialécticas}

En contraposición, cuando el docente no crea escenarios adecuados para la construcción del conocimiento, aparece otra categoría de interacciones. Estas a las que llamaremos no dialécticas, son provocadas por el docente, en general para conocer si los alumnos entendieron el conocimiento enseñado, y están caracterizadas por unidades de diálogo no relacionadas entre sí, formadas por una pregunta y una respuesta. Este patrón pregunta-respuesta se repite en diferentes momentos de una clase.

\section{Factores que influyen en las interacciones}

Hemos identificado algunos factores que inciden en la enseñanza por videoconferencia. Ellos son:

\section{Semiológicos}

La semiología o semiótica (de semeion, signo) trata los sistemas de signos y los mensajes que ellos transmiten en forma de significado (imagen mental) y significante (imagen visual). Dentro de los aspectos semiológicos, lo gestual puede incidir en las interacciones didácticas en tanto el docente esté atento a su aparición. "En una clase se va recogiendo una cantidad de información desde lo semiológico: una mirada, un tomarse la cabeza, un bostezo, un comentario oportuno. Esa información es importante para tomar decisiones, coordinar la actividad técnica y la exposición de saberes y contenidos " (EN/P3/ 4). Lo gestual es considerado tanto por los docentes como por los alumnos, muy valioso para la retroalimentación y como apoyo en el discurso. La VC limita la percepción de lo gestual debido a la falta de nitidez de la imagen, a las escasas posibilidades de movimiento, y en consecuencia, a la incorrecta interpretación de los códigos que podamos hacer.

\section{Afectivos}

Son aquellos que permiten la creación de un clima informal, un ambiente agradable cuya finalidad es dar confianza al alumno y propiciar así, la aparición de interacciones didácticas. Son intervenciones que tienen un profundo sentido empático facilitador del aprendizaje. En la videoconferencia, aquellos docentes que tienen en cuenta los aspectos afectivos crean un clima que favorece la participación de los alumnos. Esto se pudo apreciar claramente en las observaciones a las clases por video y en los sitios. Por otra parte hemos observado que aquellos docentes que no tienen en cuenta los aspectos afectivos, no favorecen la participación de los alumnos y en consecuencia las interacciones, sean éstas didácticas o no.

50 - Universidad ORT Uruguay 


\section{Tecnológicos}

La capacidad de transmisión, dada por el ancho de banda, a través de una serie de complejos procesos, permite la transmisión de señales de audio y video con algunas limitaciones técnicas. Los movimientos rápidos frecuentemente provocan una imagen poco nítida y el sonido tarda algunas décimas de segundo en llegar de un sitio a otro. La relación didáctica se ve afectada frecuentemente por la tecnología y depende de diversos elementos como la conexión, la calidad de la imagen y del audio, la cantidad de sedes conectadas, entre otros.

\section{Prácticas pedagógicas}

Las prácticas pedagógicas están relacionadas con el quehacer docente en el aula; son observables en las distintas intervenciones del profesor, en la acción que desarrolla en la clase y a través de la cual procura el aprendizaje de sus alumnos. En consecuencia, dichas prácticas están vinculadas a las metodologías y técnicas que desarrolla el docente en forma explícita o implícita, en un medio tecnológico que exige por lo menos una revisión y análisis sobre la adecuación y pertinencia de las mismas. En las clases analizadas pueden observarse las dificultades que plantean algunos docentes cuando intentan recrear en este medio algunas de las técnicas utilizadas en la clase presencial. Por otra parte, perciben la necesidad de una mayor participación de los alumnos, aunque sus prácticas sean más bien discursivas, con una mayor frecuencia de interacciones no dialécticas. No obstante, existen docentes que, en la videoconferencia, claramente fomentan las interacciones dialécticas y encuentran recursos, o bien ensayan entre distintas técnicas, logrando provocarlas tanto en cantidad como en calidad, aunque la dinámica es siempre menor a la de la clase presencial.

\section{Conclusiones}

Las clases por VC tienen características propias que inciden en los profesores, los alumnos y en los procesos de enseñanza y de aprendizaje, y que las diferencian por lo tanto de las clases presenciales. Por ello, un docente que trabaja en $\mathrm{EaD}$ debe tener siempre en cuenta, que la forma de presentar los contenidos de su curso debe adecuarse al medio que estará utilizando, y que la preparación del material didáctico para los alumnos será distinta según el medio, al igual que será diferente si se trata de una clase presencial.

La videoconferencia y la clase presencial. La producción y ocurrencia de las interacciones didácticas en los cursos por VC analizados, guardan similitud en la percepción de los profesores entrevistados, con su experiencia docente en los cursos presenciales. La videoconferencia permite que se cumplan las características propias de la interacción como la bidireccionalidad, reciprocidad e influencia mutua en la comunicación, así como aquellas referidas a las interacciones didácticas, específicamente: las interacciones dialécticas. No obstante su dinámica se ve enlentecida por la limitación del medio tecnológico empleado, en lo que se refiere al audio, a la cantidad de sedes, caídas del sistema, entre otros.

Las interacciones y el aprendizaje en la videoconferencia. Las interacciones didácticas, en particular las dialécticas, tienen posibilidades reales de ser trabajadas en el contexto de la VC, constituyéndose en una valiosa herramienta pedagógica, tal como se ha observado en las prácticas pedagógicas de algunos docentes. Existen distintos factores que pueden favorecerlas como un buen relacionamiento afectivo, y ciertas limitaciones semiológicas y tecnológicas, que adecuadamente manejadas por el docente no son obstáculos para su generación. Es el docente quien debe crear el escenario adecuado para su aparición, y luego conducir el proceso de enseñanza para que las interacciones didácticas se desarrollen. 
Capítulo 4 Las interacciones didácticas en el marco de las prácticas educativas en la videoconferencia

EI docente y la videoconferencia. La inseguridad y temor ante este nuevo medio de enseñanza y la adecuación que necesariamente los docentes deben hacer de sus prácticas pedagógicas, influyen en el grado de participación que esperan y logran de sus alumnos. El conocimiento por parte de los profesores de diferentes metodologías, técnicas y estrategias de enseñanza, les facilita y provee de más recursos para la adaptación de sus prácticas pedagógicas en la $\mathrm{VC}$, lo que no constituye un proceso fácil. El conocimiento previo del medio incidirá en su práctica facilitándola, disminuyendo la sensación de inseguridad y el nerviosismo manifestado por los docentes, frente al uso de esta tecnología.

Las prácticas pedagógicas. Por un lado, existen diferencias en las prácticas pedagógicas usadas por los docentes en la VC, que están muy relacionadas con el tipo de curso que dan. En segundo lugar, la utilización de recursos didácticos como el pizarrón, el retroproyector, etc., que se presenta en la clase presencial, no se da del mismo modo en el entorno de la videoconferencia. Así encontramos docentes que se sienten limitados por este medio tecnológico, justificando de esta forma la elección de una determinada práctica pedagógica, mientras que otros hacen una utilización estratégica de los recursos didácticos, seleccionando aquellas técnicas y estrategias más adecuadas a su materia y a este medio. Asimismo han debido hacer cambios metodológicos importantes por diversas razones, como la lentitud en la dinámica de la clase, que obliga a jerarquizar los contenidos temáticos, separar lo esencial de lo accesorio, ampliar el tiempo dedicado a aclarar dudas, responder preguntas, etc. También deben adaptar el uso que hacen del pizarrón y de otros materiales, a esta nueva herramienta. Realmente se aprecia la dificultad que sienten los docentes acostumbrados a apoyarse en el pizarrón y organizarse disponiendo de una gran superficie, pues en la $\mathrm{VC}$ deben cambiar completamente su presentación adaptándola a un espacio un poco más pequeño que una hoja tamaño carta. $\mathrm{La} \mathrm{VC}$ se presenta entonces, como un medio tecnológico que requiere una adaptación de las prácticas pedagógicas utilizadas en la enseñanza presencial.

Las interacciones didácticas y las prácticas pedagógicas. Las prácticas pedagógicas que imprimen un mayor ritmo a la clase con gran participación de los estudiantes, fomentando así las interacciones didácticas, pueden ser aplicadas en los cursos por VC. Recursos metodológicos como talleres, diálogos socráticos, dramatizaciones, dinámicas vivenciales, metodología de casos, entre otros, pueden ser desarrollados con éxito en el marco de la videoconferencia.

De acuerdo con los objetivos propuestos se logró identificar las características de las interacciones didácticas que se producen en la VC, expresadas a través de las interacciones dialécticas y no dialécticas. Si bien se observaron algunas prácticas pedagógicas que favorecen las interacciones didácticas, éste es un tema que debería ser investigado más profundamente, en cuanto a lo relacionado con la formación docente y con el conocimiento previo del medio, entre otros, como así también conocer más aún las bondades tecnológicas de esta herramienta en la enseñanza.

\section{Bibliografía}

Bengoa, C.; González, Z. 1998 Algunas consideraciones sobre el docente ante el incremento del conocimiento a fines del siglo XX. Publicación $\mathrm{N}^{\circ} 3$ del Centro de Estudios Jean Piaget. Montevideo.

Coll, C. 1994 Aprendizaje escolar y construcción del conocimiento. Buenos Aires: Paidós.

Galindo, E. La videoconferencia interactiva. Boletín electrónico Redec $\mathrm{N}^{\mathrm{o}} 2$ (tomado de: http:// pompeya.cuaed.unam.mx/areas/cuaed/educon/boletin.html). México.

GATE (Gabinete de Tele-Educación) 1998 Informe sobre tele-educación en la formación de postgrado. Madrid: Universidad Politécnica de Madrid.

Latchem, C. 1995 See what I mean? Where compressed digital videoconferencing works. En Lockwood (ed) 1995:98-107. Londres: Routledge.

Medina Rivilla, A. 1989 Didáctica e interacción en el aula. 2a . ed. Bogotá: Cincel Kapelusz.

52 - Universidad ORT Uruguay 
$\underline{\text { Capítulo } 4 \text { Las interacciones didácticas en el marco de las prácticas educativas en la videoconferencia }}$

Postic, M. 1982 La relación educativa. Madrid: Narcea.

Serrano, C. 1998 La coevaluación como proceso formativo indispensable en Educación a Distancia. Ponencia del Seminario de Educación a Distancia para América Latina y el Caribe, México.

Tiffin, J. y Rajassingham, L. 1995 In search of the virtual class. Londres: Routledge.

Universidad de Indiana, 1996 Distance learning a guide to system planning and implementation. Universidad de Indiana.

* Coordinador, Educación Externa, Universidad ORT Uruguay.

**, ***, **** Diploma en Educación, Universidad ORT Uruguay. 